ESAIM: M2AN 48 (2014) 955-968

DOI: $10.1051 / \mathrm{m} 2 \mathrm{an} / 2013129$
ESAIM: Mathematical Modelling and Numerical Analysis

www.esaim-m2an.org

\title{
A NEW QUADRILATERAL MINI-ELEMENT FOR STOKES EQUATIONS
}

\author{
Oh-In Kwon ${ }^{1}$ And Chunjae Park ${ }^{1}$
}

\begin{abstract}
We introduce a new stable MINI-element pair for incompressible Stokes equations on quadrilateral meshes, which uses the smallest number of bubbles for the velocity. The pressure is discretized with the $P_{1}$-midpoint-edge-continuous elements and each component of the velocity field is done with the standard $\boldsymbol{Q}_{1}$-conforming elements enriched by one bubble a quadrilateral. The superconvergence in the pressure of the proposed pair is analyzed on uniform rectangular meshes, and tested numerically on uniform and non-uniform meshes.
\end{abstract}

Mathematics Subject Classification. 65N30, 74S05, 76M10.

Received May 7, 2012. Revised August 5, 2013.

Published online June 30, 2014.

\section{INTRODUCTION}

In the finite element methods for incompressible Stokes problems, the pair of discrete velocity and pressure should fulfill the inf-sup condition for the stability, which is attributed to the analysis of Babuška [2] and Brezzi [4]. On triangular meshes, the MINI-element pair [1] of " $\left[P_{1} \text {-conforming }+ \text { bubble }\right]^{2} \times P_{1}$-continuous" is one of the stable pairs of the lowest order, since the simplest pair of " $\left[P_{1} \text {-conforming }\right]^{2} \times P_{0}$ " is not stable.

The several pairs of "([ $\mathbf{Q}_{1}$-conforming + bubble $]^{2}+$ bubble $) \times \mathbf{Q}_{1}$-continuous" have been suggested as the MINI-element on quadrilateral meshes [3,7]. Their discrete velocities are the standard conforming spaces enriched by at least three bubbles a quadrilateral, while the triangular MINI-element is done by two bubbles a triangle.

In this paper, we will introduce a new stable MINI-element pair on quadrilateral meshes which is " $\left[\mathbf{Q}_{1} \text {-conforming }+ \text { bubble }\right]^{2} \times P_{1}$-midpoint-edge-continuous". Compared to other quadrilateral MINI-elements, the discrete pressure has continuous averages over edges instead of the pointwise continuity. The number of bubbles for the velocity is then reduced to two a quadrilateral, similar to the triangular MINI-element. The proposed MINI-element pair is interpreted as a subspace of the stable " $\left[\mathbf{Q}_{2} \text {-conforming }\right]^{2} \times$ piecewise $P_{1}$ " on quadrilateral meshes.

Regarding the finite element solutions with the MINI-elements, although the order of convergence in pressure is analyzed to be $O(h)$ by the standard error analysis, the superconvergence of order $O\left(h^{3 / 2}\right)$ is fully understood on three-directional triangular meshes with the aid of the stabilized formulation [6]. We will analyze the similar superconvergence for the pressure of the proposed MINI-element on uniform rectangular meshes, as well as numerical tests on both uniform and non-uniform meshes.

In the following section, our MINI-element will be defined and proven to be stable through the inf-sup condition. Section 3 will be assigned for the superconvergence in pressure, which consists of three subsections

Keywords and phrases. MINI-element, superconvergence.

1 Department of Mathematics, Konkuk University, 143-701 Seoul, Korea. cjpark@konkuk.ac.kr 
for the stabilized formulation of the finite element method, the supercloseness of the bilinear interpolation and the main proof of the superconvergence, respectively. The paper will be closed with the numerical results in the final section.

Throughout the paper, standard notations for Sobolev spaces are employed and $L_{0}^{2}(\Omega)$ denotes the space of all functions in $L^{2}(\Omega)$ whose average over $\Omega$ vanish. For a bounded set $S \subset \mathbb{R}^{2}$, with its boundary $\partial S,\|\cdots\|_{m, S}$ and $|\cdots|_{m, S}$ will mean the norm and seminorm for $H^{m}(S)$. We will denote by $(\cdot, \cdot)_{S}$ and $\left.<\cdot, \cdot\right\rangle_{\partial S}$ the $L^{2}(S)$ and $L^{2}(\partial S)$ inner products, respectively. If $S=\Omega$ or $m=0$, they may be omitted in the indices.

\section{A new Quadrilateral Mini-element}

Let $\Omega$ be a simply connected polygonal domain and $\left\{\mathcal{T}_{h}\right\}_{h>0}$ a regular family of triangulations of $\Omega$ which consist of convex quadrilaterals. In $\mathcal{T}_{h}, h$ is proportional to the maximum of diameters of quadrilaterals in $\mathcal{T}_{h}$.

\section{1. $P_{1}$-midpoint-edge-continuous quadrilateral pressure}

Let $P_{h}$ be a space of piecewise linear functions as follows:

$$
P_{h}=\left\{\left.q_{h} \in L^{2}(\Omega) \quad q_{h}\right|_{Q} \in \operatorname{span}\{1, x, y\} \text { for all quadrilaterals } Q \in \mathcal{T}_{h}\right\},
$$

then the $P_{1}$-midpoint-edge-continuous quadrilateral finite element space [9] is defined by

$$
\mathcal{N} \mathcal{C}^{h}(\Omega)=\left\{q_{h} \in P_{h} \quad q_{h} \text { is continuous at every midpoints of edges in } \mathcal{T}_{h}\right\}
$$

For each vertex $\mathbf{V}$ in $\mathcal{T}_{h}$, a function $\psi^{\mathbf{V}} \in \mathcal{N C}^{h}(\Omega)$ is defined by its values at all midpoints $m$ of edges in $\mathcal{T}_{h}$ such that

$$
\psi^{\mathbf{V}}(m)= \begin{cases}1, & \text { if } m \text { belongs to an edge which meets } \mathbf{V} \\ 0, & \text { otherwise. }\end{cases}
$$

Then, if we fix an arbitrary vertex $\mathbf{V}_{0}$ in $\mathcal{T}_{h}$, the following set is a basis for $\mathcal{N C}^{h}(\Omega)$,

$$
\left\{\psi^{\mathbf{V}} \in \mathcal{N} \mathcal{C}^{h}(\Omega) \quad \mathbf{V} \text { is a vertex in } \mathcal{T}_{h}, \mathbf{V} \neq \mathbf{V}_{0}\right\}
$$

Hence, the dimension of $\mathcal{N} \mathcal{C}^{h}(\Omega)$ is the number of vertices in $\mathcal{T}_{h}$ subtracted by one.

Define an interpolation $\pi_{h}: H^{1}(\Omega) \cap C(\Omega) \rightarrow \mathcal{N C}^{h}(\Omega)$ as

$$
\pi_{h} v=\sum_{\mathbf{V}} \frac{v(\mathbf{V})}{2} \psi^{\mathbf{V}}, \quad \forall v \in H^{1}(\Omega) \cap C(\Omega),
$$

where the summation runs over all interior vertices $\mathbf{V}$ in $\mathcal{T}_{h}$. Then, the interpolation error is estimated by the following, for $v \in H^{2}(\Omega)$,

$$
\left\|v-\pi_{h} v\right\|_{0}+h\left(\sum_{Q \in \mathcal{T}_{h}}\left|v-\pi_{h} v\right|_{1, Q}^{2}\right)^{1 / 2} \leq C h^{2}|v|_{2} .
$$

It is noted that $\mathcal{N C}^{h}(\Omega)$ is a common subspace of other low order nonconforming spaces such as the Rotated $Q_{1}$ or DSSY finite element spaces $[5,10]$. 


\subsection{A new MINI-element pair}

Let $\widehat{Q}=[-1,1]^{2}$ be a reference rectangle and $\widehat{\mathbf{Q}}_{1}, \widehat{\mathbf{Q}}_{2}$ bilinear and biquadratic polynomial spaces on $\widehat{Q}$, respectively, that is,

$$
\widehat{\mathbf{Q}}_{1}=\operatorname{span}\{1, \widehat{x}, \widehat{y}, \widehat{x} \widehat{y}\}, \quad \widehat{\mathbf{Q}}_{2}=\widehat{\mathbf{Q}}_{1} \bigoplus \operatorname{span}\left\{\widehat{x}^{2}, \widehat{y}^{2}, \widehat{x}^{2} \widehat{y}, \widehat{x} \widehat{y}^{2}, \widehat{x}^{2} \widehat{y}^{2}\right\} .
$$

For each quadrilateral $Q \in \mathcal{T}_{h}$, the canonical bijective bilinear map from $\widehat{Q}$ to $Q$ is denoted by $F_{Q}$ and function spaces $\mathbf{Q}_{1}(Q), \mathbf{Q}_{2}(Q)$ on $Q$ are defined as follows:

$$
\mathbf{Q}_{k}(Q)=\left\{\widehat{v} \circ F_{Q}^{-1} \widehat{v} \in \widehat{\mathbf{Q}}_{k}\right\}, \quad k=1,2 .
$$

Then, the standard conforming finite element spaces $\mathbf{Q}_{1, h}, \mathbf{Q}_{2, h}$ over $\mathcal{T}_{h}$ are defined by

$$
\mathbf{Q}_{k, h}=\left\{\left.v_{h} \in C(\Omega) \quad v_{h}\right|_{Q} \in \mathbf{Q}_{k}(Q) \text { for all quadrilaterals } Q \in \mathcal{T}_{h}\right\},
$$

as well as $\mathbf{Q}_{k, h, 0}=\mathbf{Q}_{k, h} \cap H_{0}^{1}(\Omega)$ for $k=1,2$.

It is well-known that the pair of $\left[\mathbf{Q}_{2, h, 0}\right]^{2}$ for velocity and $P_{h} \cap L_{0}^{2}(\Omega)$ for pressure satisfy the inf-sup condition for Stokes equations in the following proposition, whose proof is referred to Section 3.2, Chapter II in [8].

Proposition 2.1. Let $p_{h} \in P_{h} \cap L_{0}^{2}(\Omega)$. There exists $\mathbf{v}_{h} \in\left[\mathbf{Q}_{2, h, 0}\right]^{2}$ such that

$$
\int_{\Omega} p_{h} \operatorname{div} \mathbf{v}_{h} \mathrm{~d} s=\left\|p_{h}\right\|_{0, \Omega}^{2}, \quad\left|\mathbf{v}_{h}\right|_{1, \Omega} \leq \frac{1}{\beta}\left\|p_{h}\right\|_{0, \Omega},
$$

for a positive constant $\beta$ which depends only on $\Omega$.

In order to introduce a MINI-element pair, we choose a bubble function $b_{Q} \in \mathbf{Q}_{2}(Q)$ for each quadrilateral $Q \in \mathcal{T}_{h}$ such that

$$
b_{Q} \circ F_{Q}=\left(\widehat{x}^{2}-1\right)\left(\widehat{y}^{2}-1\right) \in \widehat{\mathbf{Q}}_{2},
$$

which vanishes on the boundary of $Q$. Then, identifying $b_{Q}$ with its trivial extension into $C(\Omega)$, we propose a new MINI-element pair for Stokes equations as follows:

$$
X_{h}=\left[\mathbf{Q}_{1, h, 0} \bigoplus_{Q \in \mathcal{T}_{h}} \operatorname{span}\left\{b_{Q}\right\}\right]^{2}, \quad M_{h}=\mathcal{N} \mathcal{C}^{h}(\Omega) \cap L_{0}^{2}(\Omega) .
$$

The pair $X_{h} \times M_{h}$ turns out to be stable to solve Stokes equations in the following theorem.

Theorem 2.2 (Inf-sup condition). Let $\mu_{h} \in M_{h}$. There exists $\mathbf{v}_{h} \in X_{h}$ such that

$$
\int_{\Omega} \mu_{h} \operatorname{div} \mathbf{v}_{h} \mathrm{~d} s=\left\|\mu_{h}\right\|_{0, \Omega}^{2}, \quad\left|\mathbf{v}_{h}\right|_{1, \Omega} \leq \frac{1}{\beta}\left\|\mu_{h}\right\|_{0, \Omega},
$$

for a positive constant $\beta$ which depends only on $\Omega$.

Proof. Define an interpolation operator $\Pi_{h}: \mathbf{Q}_{2, h, 0} \rightarrow \mathbf{Q}_{1, h, 0} \bigoplus_{Q \in \mathcal{T}_{h}} \operatorname{span}\left\{b_{Q}\right\}$ such that

$$
\Pi_{h} v(\mathbf{V})=v(\mathbf{V}), \quad \int_{Q} \Pi_{h} v \mathrm{~d} s=\int_{Q} v \mathrm{~d} s, \quad \forall v \in \mathbf{Q}_{2, h, 0},
$$

for all vertices $\mathbf{V}$ and quadrilaterals $Q$ in $\mathcal{T}_{h}$. Then, by Bramble lemma, we have

$$
\left|v-\Pi_{h} v\right|_{1, Q} \leq C h|v|_{2, Q}, \quad \text { for all quadrilaterals } Q \in \mathcal{T}_{h},
$$

with some constant $C$ regardless of $v, Q$ and $h$. 
Applying the argument of Lemma A.6, Appendix A in [8] for triangles into quadrilaterals, the following inverse inequality is established with some constant $C$,

$$
|v|_{2, Q} \leq C h^{-1}|v|_{1, Q} .
$$

From (2.5) and (2.6), the operator $\Pi_{h}$ is bounded by

$$
\left|\Pi_{h} v\right|_{1, \Omega} \leq C|v|_{1, \Omega}, \quad \forall v \in \mathbf{Q}_{2, h, 0} .
$$

Now, if $\mu_{h} \in M_{h}$, by Proposition 2.1, there exists $\mathbf{w}_{h} \in\left[\mathbf{Q}_{2, h, 0}\right]^{2}$ such that

$$
\int_{\Omega} \mu_{h} \operatorname{div} \mathbf{w}_{h} \mathrm{~d} s=\left\|\mu_{h}\right\|_{0, \Omega}^{2}, \quad\left|\mathbf{w}_{h}\right|_{1, \Omega} \leq \frac{1}{\beta}\left\|\mu_{h}\right\|_{0, \Omega},
$$

for a positive constant $\beta$ which depends only on $\Omega$. For $\mathbf{w}_{h}=\left(w_{1}, w_{2}\right)$, define $\mathbf{v}_{h} \in X_{h}$ by

$$
\mathbf{v}_{h}=\left(\Pi_{h} w_{1}, \Pi_{h} w_{2}\right) .
$$

Then, it will be shown

$$
\int_{\Omega} \mu_{h} \operatorname{div} \mathbf{w}_{h} \mathrm{~d} s=\int_{\Omega} \mu_{h} \operatorname{div} \mathbf{v}_{h} \mathrm{~d} s .
$$

We have, by integration by parts,

$$
\int_{\Omega} \mu_{h} \operatorname{div}\left(\mathbf{w}_{h}-\mathbf{v}_{h}\right) \mathrm{d} s=\sum_{Q \in \mathcal{T}_{h}} \int_{\partial Q} \mu_{h}\left(\mathbf{w}_{h}-\mathbf{v}_{h}\right) \cdot \mathbf{n} \mathrm{d} l-\sum_{Q \in \mathcal{T}_{h}} \int_{Q} \nabla \mu_{h} \cdot\left(\mathbf{w}_{h}-\mathbf{v}_{h}\right) \mathrm{d} s
$$

where $\mathbf{n}$ is the unit vector which is outward normal to $\partial Q$. Since $\nabla \mu_{h}$ is a constant vector, by definition of $\Pi_{h}$ in (2.4), the second term in the right hand side of (2.10) is null.

Denote by $m_{E}$ the midpoint of edge $E \in \mathcal{T}_{h}$. Since $\mathbf{w}_{h}-\mathbf{v}_{h}$ is continuous on interior edges and vanishes on boundary edges, the first term is rewritten as:

$$
\sum_{Q \in \mathcal{T}_{h}} \int_{\partial Q} \mu_{h}\left(\mathbf{w}_{h}-\mathbf{v}_{h}\right) \cdot \mathbf{n} \mathrm{d} l=\sum_{Q \in \mathcal{T}_{h}} \sum_{E \in \partial Q} \int_{E}\left(\mu_{h}-\mu_{h}\left(m_{E}\right)\right)\left(\mathbf{w}_{h}-\mathbf{v}_{h}\right) \cdot \mathbf{n} \mathrm{d} l .
$$

We note that $\mu_{h}-\mu_{h}\left(m_{E}\right)$ and $\mathbf{w}_{h}-\mathbf{v}_{h}$ are the linear and quadratic functions on $E$ vanishing at the midpoint and two endpoints of $E$, respectively. Thus, all integrals over edges in (2.11) vanish, since the integrands are odd and cubic.

This means (2.9) and completes the proof with (2.7), (2.8).

Let $(\mathbf{u}, p) \in\left[H_{0}^{1}(\Omega)\right]^{2} \times L_{0}^{2}(\Omega)$ be the solution of the variational form of a Stokes equation such that, for a source function $\mathbf{f} \in\left[L^{2}(\Omega)\right]^{2}$,

$$
(\nabla \mathbf{u}, \nabla \mathbf{v})-(p, \operatorname{div} \mathbf{v})+(q, \operatorname{div} \mathbf{u})=(\mathbf{f}, \mathbf{v}), \quad \forall(\mathbf{v}, q) \in\left[H_{0}^{1}(\Omega)\right]^{2} \times L_{0}^{2}(\Omega),
$$

and $\left(\mathbf{u}_{h}, p_{h}\right) \in X_{h} \times M_{h}$ be the solution of the following discrete variational problem,

$$
\left(\nabla \mathbf{u}_{h}, \nabla \mathbf{v}_{h}\right)-\left(p_{h}, \operatorname{div} \mathbf{v}_{h}\right)+\left(q_{h}, \operatorname{div} \mathbf{u}_{h}\right)=\left(\mathbf{f}, \mathbf{v}_{h}\right), \quad \forall\left(\mathbf{v}_{h}, q_{h}\right) \in X_{h} \times M_{h} .
$$

If $(\mathbf{u}, p)$ belongs to $\left[H^{2}(\Omega)\right]^{2} \times H^{1}(\Omega)$, through the inf-sup condition in Theorem 2.2, the approximation error is estimated by

$$
\left|\mathbf{v}-\mathbf{v}_{h}\right|_{1}+\left\|p-p_{h}\right\|_{0} \leq C h\left(|\mathbf{v}|_{2}+|p|_{1}\right)
$$

for a constant $C$ which depends only on $\Omega$ and the regularity of $\mathcal{T}_{h}$.

While the order of convergence in pressure is $O(h)$ as in (2.14) on general quadrilateral meshes, the remaining of the paper will be devoted to the analysis of the superconvergence of order $O\left(h^{3 / 2}\right)$ on uniform rectangular meshes. 


\section{Superconvergence in Pressure}

The superconvergence in pressure is well understood [6] for the MINI-element of Arnold et al. [1] on threedirectional triangular meshes. In this section, following the arguments in [6] with the attention on the midpointedge-continuous pressure instead of the continuous one, we will prove that the proposed MINI-element has the same property. Throughout the section, we assume that $\mathcal{T}_{h}$ consists of uniform rectangles, whose edges are all parallel to the coordinate axes.

For a function $\mathbf{v}_{h}$ in $X_{h}$, the bilinear and bubble parts of $\mathbf{v}_{h}$ are denoted by $\mathbf{v}_{L}, \mathbf{v}_{b}$, respectively, so that:

$$
\mathbf{v}_{h}=\mathbf{v}_{L}+\mathbf{v}_{b}, \quad \mathbf{v}_{L} \in\left[\mathbf{Q}_{1, h, 0}\right]^{2},\left.\quad \mathbf{v}_{b}\right|_{Q} \in\left[\operatorname{span}\left\{b_{Q}\right\}\right]^{2} \text { for all } Q \in \mathcal{T}_{h} .
$$

We note that they are orthogonal to each other in $H^{1}$ inner product since:

$$
\left(\nabla \mathbf{v}_{L}, \nabla \mathbf{v}_{b}\right)_{Q}=0, \quad \text { for each rectangle } Q \in \mathcal{T}_{h} .
$$

\subsection{Stabilized variational form}

We will show that if $\left(\mathbf{u}_{h}, p_{h}\right) \in X_{h} \times M_{h}$ satisfy (2.13), the pair of the bilinear part $\mathbf{u}_{L}$ of $\mathbf{u}_{h}$ and the pressure $p_{h}$ is a solution of a stabilized variational problem.

Set a bilinear form $B_{h}$ on $H_{0}^{1}(\Omega) \times L_{0}^{2}(\Omega)$ as:

$$
B_{h}(\mathbf{u}, p ; \mathbf{v}, q)=(\nabla \mathbf{u}, \nabla \mathbf{v})-(p, \operatorname{div} \mathbf{v})+(q, \operatorname{div} \mathbf{u})+\sum_{Q \in \mathcal{T}_{h}}\left(-\Delta \mathbf{u}+\nabla p, \tau_{Q} \nabla q\right)_{Q},
$$

where $\tau_{Q}$ is the bubble function $b_{Q}$ in $(2.3)$ multiplied by a constant for each rectangle $Q \in \mathcal{T}_{h}$ such that

$$
\tau_{Q}=\frac{\int_{Q} b_{Q} \mathrm{~d} s}{\int_{Q}\left|\nabla b_{Q}\right|^{2} \mathrm{~d} s} b_{Q} .
$$

We note that $\tau_{Q}$ is a nonnegative function which satisfies,

$$
\left(\nabla \tau_{Q}, \nabla \tau_{Q}\right)_{Q}=\left(\tau_{Q}, 1\right)_{Q}=\alpha^{2} h^{2}(1,1)_{Q}, \quad\left\|\tau_{Q}\right\|_{\infty, Q}=\gamma^{2} h^{2},
$$

with some fixed positive constants $\alpha$ and $\gamma$. From (3.3), we have an identity,

$$
\left\|\tau_{Q}^{1 / 2} \nabla q_{h}\right\|_{0, Q}=\alpha h\left\|\nabla q_{h}\right\|_{0, Q},
$$

for all rectangles $Q \in \mathcal{T}_{h}$ and piecewise linear pressures $q_{h} \in M_{h}$.

Lemma 3.1. If $\left(\mathbf{u}_{h}, p_{h}\right) \in X_{h} \times M_{h}$ satisfies (2.13), then $\left(\mathbf{u}_{L}, p_{h}\right) \in\left[\mathbf{Q}_{1, h, 0}\right]^{2} \times M_{h}$ does

$$
B_{h}\left(\mathbf{u}_{L}, p_{h} ; \mathbf{v}_{L}, q_{h}\right)=\left(\mathbf{f}, \mathbf{v}_{L}\right)+\sum_{Q \in \mathcal{T}_{h}}\left(\mathbf{f}, \tau_{Q} \nabla q_{h}\right)_{Q}, \quad \forall\left(\mathbf{v}_{L}, q_{h}\right) \in\left[\mathbf{Q}_{1, h, 0}\right]^{2} \times M_{h} .
$$

Proof. Let $\left(\mathbf{v}_{L}, q_{h}\right) \in\left[\mathbf{Q}_{1, h, 0}\right]^{2} \times M_{h}$, then for any bubble $\mathbf{v}_{b}$, (2.13) establishes that, from the orthogonality in $(3.1)$,

$$
\begin{aligned}
\left(\nabla \mathbf{u}_{L}, \nabla \mathbf{v}_{L}\right)-\left(p_{h}, \operatorname{div} \mathbf{v}_{L}\right)+\left(q_{h}, \operatorname{div} \mathbf{u}_{L}\right) & =\left(\mathbf{f}, \mathbf{v}_{L}+\mathbf{v}_{b}\right)-\left(\nabla \mathbf{u}_{b}, \nabla \mathbf{v}_{b}\right)+\left(p_{h}, \operatorname{div} \mathbf{v}_{b}\right)-\left(q_{h}, \operatorname{div} \mathbf{u}_{b}\right) \\
& =\left(\mathbf{f}, \mathbf{v}_{L}\right)+\sum_{Q \in \mathcal{T}_{h}}\left(\mathbf{f}-\nabla p_{h}, \mathbf{v}_{b}\right)_{Q}-\left(\nabla \mathbf{u}_{b}, \nabla \mathbf{v}_{b}\right)+\sum_{Q \in \mathcal{T}_{h}}\left(\nabla q_{h}, \mathbf{u}_{b}\right)_{Q} .
\end{aligned}
$$


We note that $\nabla q_{h}$ is piecewisely constant and $\left.\mathbf{u}_{b}\right|_{Q}=\tau_{Q}\left(c_{1}, c_{2}\right)$ for some constants $c_{1}, c_{2}$. If we choose a bubble $\mathbf{v}_{b}$ such that $\left.\mathbf{v}_{b}\right|_{Q}=\tau_{Q} \nabla q_{h}$ for each rectangle $Q \in \mathcal{T}_{h}$, then the following identity comes from (3.3),

$$
\left(\nabla \mathbf{u}_{b}, \nabla \mathbf{v}_{b}\right)=\sum_{Q \in \mathcal{T}_{h}}\left(\nabla q_{h}, \mathbf{u}_{b}\right)
$$

Thus, the last two terms in (3.6) vanish and (3.6) implies (3.5), since $\Delta \mathbf{u}_{L}=0$.

Although the bilinear form $B_{h}$ in (3.2) is not uniformly coercive on $\left[\mathbf{Q}_{1, h, 0}\right]^{2} \times M_{h}$, we have a stability for $B_{h}$ in the following lemma. Define a triple norm as

$$
\|\mid(\mathbf{v}, q)\| \|_{B}=\left(|\mathbf{v}|_{1}^{2}+\|q\|_{0}^{2}+\sum_{Q \in \mathcal{T}_{h}}\left\|\tau_{Q}^{1 / 2} \nabla q\right\|_{0, Q}^{2}\right)^{1 / 2}
$$

Lemma 3.2. If $\left(\mathbf{v}_{L}, q_{h}\right) \in\left[\mathbf{Q}_{1, h, 0}\right]^{2} \times M_{h}$, there exists $\mathbf{w}_{L} \in\left[\mathbf{Q}_{1, h, 0}\right]^{2}$ such that

$$
B_{h}\left(\mathbf{v}_{L}, q_{h} ; \mathbf{w}_{L}, q_{h}\right) \geq \beta_{B}\left\|\left|\left(\mathbf{v}_{L}, q_{h}\right)\|\|_{B}\right|\right\|\left(\mathbf{w}_{L}, q_{h}\right) \mid \|_{B},
$$

where $\beta_{B}$ is a constant which depends only on $\Omega$.

Proof. By the inf-sup condition in Theorem 2.2, there exists $\mathbf{z}_{h} \in X_{h}$ such that

$$
\left(-q_{h}, \operatorname{div} \mathbf{z}_{h}\right)=\left\|q_{h}\right\|_{0}^{2}, \quad\left|\mathbf{z}_{h}\right|_{1} \leq \frac{1}{\beta}\left\|q_{h}\right\|_{0},
$$

with some constant $\beta$ regardless of $h$.

For the bubble part $\mathbf{z}_{b}$, we expand, from (3.4),

$$
\begin{aligned}
\left|\left(q_{h}, \operatorname{div} \mathbf{z}_{b}\right)\right| & =\sum_{Q \in \mathcal{T}_{h}}\left(\nabla q_{h}, \mathbf{z}_{b}\right)_{Q} \leq \sum_{Q \in \mathcal{T}_{h}} h\left\|\nabla q_{h}\right\|_{0, Q}\left|\mathbf{z}_{b}\right|_{1, Q} \\
& =\alpha^{-1} \sum_{Q \in \mathcal{T}_{h}}\left\|\tau_{Q}^{1 / 2} \nabla q_{h}\right\|_{0, Q}\left|\mathbf{z}_{b}\right|_{1, Q} \\
& \leq \alpha^{-1}\left(\sum_{Q \in \mathcal{T}_{h}}\left\|\tau_{Q}^{1 / 2} \nabla q_{h}\right\|_{0, Q}^{2}\right)^{1 / 2}\left(\sum_{Q \in \mathcal{T}_{h}}\left|\mathbf{z}_{b}\right|_{1, Q}^{2}\right)^{1 / 2} \\
& \leq \frac{\beta^{2}}{2}\left|\mathbf{z}_{b}\right|_{1}^{2}+\frac{1}{2 \alpha^{2} \beta^{2}}\left(\sum_{Q \in \mathcal{T}_{h}}\left\|\tau_{Q}^{1 / 2} \nabla q_{h}\right\|_{0, Q}^{2}\right)
\end{aligned}
$$

as well as for the bilinear part $\mathbf{z}_{L}$,

$$
\left|\left(\nabla \mathbf{v}_{L}, \nabla \mathbf{z}_{L}\right)\right| \leq\left|\mathbf{v}_{L}\right|_{1}\left|\mathbf{z}_{L}\right|_{1} \leq \frac{\beta^{2}}{2}\left|\mathbf{z}_{L}\right|_{1}^{2}+\frac{1}{2 \beta^{2}}\left|\mathbf{v}_{L}\right|_{1}^{2} .
$$

Then, by the orthogonality (3.1), we combine (3.9)-(3.11) to get the following:

$$
B_{h}\left(\mathbf{v}_{L}, q_{h} ; \mathbf{z}_{L}, 0\right) \geq \frac{1}{2}\left\|q_{h}\right\|_{0}^{2}-\sigma\left(\left|\mathbf{v}_{L}\right|_{1}^{2}+\sum_{Q \in \mathcal{T}_{h}}\left\|\tau_{Q}^{1 / 2} \nabla q_{h}\right\|_{0, Q}^{2}\right),
$$

for a constant $\sigma=\max \left(1 /\left(2 \alpha^{2} \beta^{2}\right), 1 /\left(2 \beta^{2}\right)\right)$. 
If we set $\mathbf{w}_{L}=\mathbf{v}_{L}+2 /(1+2 \sigma) \mathbf{z}_{L}$, then, from (3.12), $\mathbf{w}_{L}$ satisfies that

$$
\begin{aligned}
B_{h}\left(\mathbf{v}_{L}, q_{h} ; \mathbf{w}_{L}, q_{h}\right) & =B_{h}\left(\mathbf{v}_{L}, q_{h} ; \mathbf{v}_{L}, q_{h}\right)+2 /(1+2 \sigma) B_{h}\left(\mathbf{v}_{L}, q_{h} ; \mathbf{z}_{L}, 0\right) \\
& \geq 1 /(1+2 \sigma)\|\|\left(\mathbf{v}_{L}, q_{h}\right) \|_{B}^{2} .
\end{aligned}
$$

From (3.9), the triple norm of $\left(\mathbf{w}_{L}, q_{h}\right)$ is bounded by

$$
\left\|\left|\left(\mathbf{w}_{L}, q_{h}\right)\right|\right\|_{B} \leq(1+2 /(\beta+2 \beta \sigma))\|\|\left(\mathbf{v}_{L}, q_{h}\right) \|_{B} .
$$

Thus, $\mathbf{w}_{L}$ satisfies $(3.8)$ for the constant $\beta_{B}=1 /(1+2 \sigma)(1+2 /(\beta+2 \beta \sigma))^{-1}$ which depends only on $\Omega$.

\subsection{Supercloseness}

Let $\widehat{Q}=[-1,1]^{2}$ be the reference rectangle and $\widehat{E}=\{(x, y) \in \widehat{Q} \quad x=1\}$ an edge of $\widehat{Q}$. If $\widehat{g} \in H^{1}(\widehat{Q})$, for a vector field $V=(x+1,0)$ on $\widehat{Q}$, we have the following:

$$
2 \int_{\widehat{E}} \widehat{g}^{2} \mathrm{~d} l=\int_{\widehat{Q}} \operatorname{div}\left(\widehat{g}^{2} V\right) \mathrm{d} s=\int_{\widehat{Q}}\left(\widehat{g}^{2}+2 \widehat{g} \nabla \widehat{g} \cdot V\right) \mathrm{d} s .
$$

Thus, if $E$ is an edge of a rectangle $Q$ in $\mathcal{T}_{h}$ and $g \in H^{1}(Q)$, then the following local trace theorem holds, with a constant $C$ which depends only the shape of $Q$,

$$
\|g\|_{0, E} \leq C\left(h^{-1}\|g\|_{0, Q}^{2}+\|g\|_{0, Q}|g|_{1, Q}\right)^{1 / 2}
$$

Especially, if $r_{h}$ is a linear function on $Q$ in $\mathcal{T}_{h}$, since $\left|r_{h}\right|_{1, Q} \leq C h^{-1}\left\|r_{h}\right\|_{0, Q}$, we have

$$
\left\|r_{h}\right\|_{0, E} \leq C h^{-1 / 2}\left\|r_{h}\right\|_{0, Q} .
$$

We will establish two superclosenesses in the following two lemmas, which are the principal ingredients for the proof of the superconvergence of the pressure $p_{h}$ in (2.13). For any continuous function $v \in C(\Omega)$, denote by $v_{I}$ the standard bilinear interpolation of $v$ into $\mathbf{Q}_{1, h}$ such that

$$
v_{I}(\mathbf{V})=v(\mathbf{V}) \quad \text { for all vertices } \mathbf{V} \text { in } \mathcal{T}_{h} .
$$

If $\mathbf{v}=\left(v_{1}, v_{2}\right) \in[C(\Omega)]^{2}$, then $\mathbf{v}_{I}=\left(v_{1, I}, v_{2, I}\right)$.

Lemma 3.3. Let $\mathbf{u} \in\left[H^{3}(\Omega)\right]^{2}$. Then, for all $\mathbf{w}_{h} \in\left[\mathbf{Q}_{1, h}\right]^{2}$,

$$
\int_{\Omega} \nabla\left(\mathbf{u}-\mathbf{u}_{I}\right) \cdot \nabla \mathbf{w}_{h} \mathrm{~d} s \leq C h^{2}|\mathbf{u}|_{3}\left|\mathbf{w}_{h}\right|_{1},
$$

where $C$ is a constant which depends only on $\Omega$.

Proof. Let $u, u_{I}, w$ be the first components of $\mathbf{u}, \mathbf{u}_{I}$ and $\mathbf{w}_{h}$, respectively. It is sufficient to prove

$$
\int_{\Omega}\left(u-u_{I}\right)_{x} w_{x} \mathrm{~d} s \leq C h^{2}|u|_{3}|w|_{1} .
$$

For each rectangle $Q$ in $\mathcal{T}_{h}$, define a linear functional $\Psi$ on $H^{3}(Q)$ by the following:

$$
\Psi(v)=\int_{Q}\left(v-v_{I}\right)_{x} w_{x} \mathrm{~d} s, \quad \forall v \in H^{3}(Q) .
$$

Then, we have

$$
|\Psi(v)| \leq C h|v|_{2, Q}|w|_{1, Q} .
$$


Since $w_{x}$ is a function of $y$, by simple calculation with the definition of $v_{I}$, we have

$$
\Psi\left(x^{2}\right)=\Psi\left(y^{2}\right)=\Psi(x y)=0 .
$$

Let $\overline{u_{x x}}, \overline{u_{x y}}, \overline{u_{y y}}$ be the respective averages of $u_{x x}, u_{x y}, u_{y y}$ over $Q$ and $\varphi$ a quadratic function in $P_{2}(Q)$ such that

$$
\varphi=\frac{1}{2}\left(\overline{u_{x x}} x^{2}+\overline{u_{y y}} y^{2}\right)+\overline{u_{x y}} x y .
$$

Then, by Poincaré Lemma, we establish

$$
|u-\varphi|_{2, Q}=\left(\left\|u_{x x}-\overline{u_{x x}}\right\|_{0, Q}^{2}+2\left\|u_{x y}-\overline{u_{x y}}\right\|_{0, Q}^{2}+\left\|u_{y y}-\overline{u_{y y}}\right\|_{0, Q}^{2}\right)^{1 / 2} \leq C h|u|_{3, Q} .
$$

From (3.16), (3.17), (3.19), we have

$$
|\Psi(u)|=|\Psi(u-\varphi)| \leq C h|u-\varphi|_{2, Q}|w|_{1, Q} \leq C h^{2}|u|_{3, Q}|w|_{1, Q},
$$

which means (3.15).

The following proposition is a frequently used characteristic for the jump of a midpoint-edge-continuous function across an edge in $\mathcal{T}_{h}$.

Proposition 3.4. Let $r_{h} \in \mathcal{N C}^{h}(\Omega)$ and $Q_{1}, Q_{2}$ be two adjacent rectangles in $\mathcal{T}_{h}$ which share an edge E. If $g \in H^{1}\left(Q_{1} \cup Q_{2}\right)$, then

$$
\left|\int_{E} g\left(\left.r_{h}\right|_{Q_{1}}-\left.r_{h}\right|_{Q_{2}}\right) \mathrm{d} l\right| \leq C \sum_{k=1}^{2}|g|_{1, Q_{k}}\left\|\tau_{Q}^{1 / 2} \nabla r_{h}\right\|_{0, Q_{k}}
$$

where $C$ is a constant which depends only the shape of rectangles in $\mathcal{T}_{h}$.

Proof. Since $r_{h}$ is continuous at the midpoint of $E$, there is an average $\overline{r_{h}}$ of $r_{h}$ over $E$ such that

$$
\left.\int_{E} r_{h}\right|_{Q_{1}} \mathrm{~d} l=\left.\int_{E} r_{h}\right|_{Q_{2}} \mathrm{~d} l=\int_{E} \overline{r_{h}} \mathrm{~d} l
$$

We split the left hand side of (3.20) into

$$
\left|\int_{E} g\left(\left.r_{h}\right|_{Q_{1}}-\left.r_{h}\right|_{Q_{2}}\right) \mathrm{d} l\right| \leq\left|\int_{E} g\left(\left.r_{h}\right|_{Q_{1}}-\overline{r_{h}}\right) \mathrm{d} l\right|+\left|\int_{E} g\left(\left.r_{h}\right|_{Q_{2}}-\overline{r_{h}}\right) \mathrm{d} l\right| .
$$

For each $k=1,2$, denote by $\overline{g_{k}}$ the average of $g$ over $Q_{k}$, then we have, from (3.4) and the local trace Theorem (3.13),

$$
\begin{aligned}
\left|\int_{E} g\left(\left.r_{h}\right|_{Q_{k}}-\overline{r_{h}}\right) \mathrm{d} l\right| & =\left|\int_{E}\left(g-\overline{g_{k}}\right)\left(\left.r_{h}\right|_{Q_{k}}-\overline{r_{h}}\right) \mathrm{d} l\right| \leq\left\|g-\overline{g_{k}}\right\|_{0, E}\left\|\left.r_{h}\right|_{Q_{k}}-\overline{r_{h}}\right\|_{0, E} \\
& \leq C h^{1 / 2}|g|_{1, Q_{k}} h^{1 / 2}\left|r_{h}\right|_{1, Q_{k}} \leq C|g|_{1, Q_{k}}\left\|\tau_{Q}^{1 / 2} \nabla r_{h}\right\|_{0, Q_{k}},
\end{aligned}
$$

where $C$ is a constant which depends only on the shape of $Q_{k}$. It means (3.20).

Lemma 3.5. Let $\mathbf{u} \in\left[H^{3}(\Omega)\right]^{2}$. Then, for all $r_{h} \in M_{h}$,

$$
\int_{\Omega} r_{h} \operatorname{div}\left(\mathbf{u}-\mathbf{u}_{I}\right) \mathrm{d} s \leq C h^{3 / 2}\|\mathbf{u}\|_{3}\left(\left\|r_{h}\right\|_{0}^{2}+\sum_{Q \in \mathcal{T}_{h}}\left\|\tau_{Q}^{1 / 2} \nabla r_{h}\right\|_{0, Q}^{2}\right)^{1 / 2}
$$

where $C$ is a constant which depends only on $\Omega$. 
Proof. Let $u, u_{I}$ be the first components of $\mathbf{u}, \mathbf{u}_{I}$, respectively. It is enough to show that

$$
\int_{\Omega} r_{h}\left(u-u_{I}\right)_{x} \mathrm{~d} s \leq C h^{3 / 2}\|u\|_{3}\left(\left\|r_{h}\right\|_{0}^{2}+\sum_{Q \in \mathcal{T}_{h}}\left\|\tau_{Q}^{1 / 2} \nabla r_{h}\right\|_{0, Q}^{2}\right)^{1 / 2} .
$$

For each rectangle $Q$ in $\mathcal{T}_{h}$ of width $h$, define a linear functional $\Psi$ on $H^{3}(Q)$ by

$$
\Psi(v)=\int_{Q}\left(r_{h}\left(v-v_{I}\right)_{x}-\frac{h^{2}}{12}\left(v_{x x} r_{h}\right)_{x}\right) \mathrm{d} s, \quad \forall v \in H^{3}(Q) .
$$

Since $\left|r_{h}\right|_{1, Q} \leq C h^{-1}\left\|r_{h}\right\|_{0, Q}$, we estimate $|\Psi(v)|$ as

$$
\begin{aligned}
|\Psi(v)| & \leq C h\left\|r_{h}\right\|_{0, Q}|v|_{2, Q}+\frac{h^{2}}{12}\left(|v|_{3, Q}\left\|r_{h}\right\|_{0, Q}+|v|_{2, Q}\left|r_{h}\right|_{1, Q}\right) \\
& \leq C h\left\|r_{h}\right\|_{0, Q}\left(|v|_{2, Q}+h|v|_{3, Q}\right) .
\end{aligned}
$$

We note that the integrand in (3.22) vanishes, if $v$ belongs to $\operatorname{span}\left\{1, x, y, x y, y^{2}\right\}$. By definition of $v_{I}$, we have

$$
\Psi\left(x^{2}\right)=2 \int_{Q}\left(r_{h}(x-\alpha)-\frac{h^{2}}{12}\left(r_{h}\right)_{x}\right) \mathrm{d} s
$$

where $\alpha$ is the $x$-coordinate of the center of $Q$. Since the integral in (3.24) vanishes for the linear function $r_{h}$, $\Psi(\varphi)=0$ for all quadratic functions $\varphi \in P_{2}(Q)$. Then, by similar arguments in (3.18) and (3.19), the following estimation is obtained from (3.23a):

$$
|\Psi(u)|=|\Psi(u-\varphi)| \leq C h\left\|r_{h}\right\|_{0, Q}\left(|u-\varphi|_{2, Q}+h|u|_{3, Q}\right) \leq C h^{2}\left\|r_{h}\right\|_{0, Q}|u|_{3, Q} .
$$

Next, the second term in the integrand for $\Psi(u)$ in (3.22) satisfies:

$$
\begin{aligned}
\sum_{Q \in \mathcal{T}_{h}} \int_{Q}\left(u_{x x} r_{h}\right)_{x} \mathrm{~d} s & =\sum_{Q \in \mathcal{T}_{h}} \int_{\partial Q} u_{x x} r_{h} \mathbf{n}_{x} \mathrm{~d} l \\
& =\sum_{E} \int_{E} u_{x x}\left(\left.r_{h}\right|_{Q_{L}}-\left.r_{h}\right|_{Q_{R}}\right) \mathrm{d} l+\int_{\partial \Omega} u_{x x} r_{h} \mathbf{n}_{x} \mathrm{~d} l
\end{aligned}
$$

where $E$ runs over all interior vertical edges and $Q_{L}$ shares $E$ with $Q_{R}$ in its right side.

By Proposition 3.4 above, the first term in (3.26a) and (3.26b) is estimated by

$$
\left|\sum_{E} \int_{E} u_{x x}\left(\left.r_{h}\right|_{Q_{L}}-\left.r_{h}\right|_{Q_{R}}\right) \mathrm{d} l\right| \leq C|u|_{3, \Omega}\left(\sum_{Q \in \mathcal{T}_{h}}\left\|\tau_{Q}^{1 / 2} \nabla r_{h}\right\|_{0, Q}^{2}\right)^{1 / 2} .
$$

From the global trace theorem for $u_{x x}$ and the local one for $r_{h}$ in (3.14), the second term is done as

$$
\left|\int_{\partial \Omega} u_{x x} r_{h} \mathbf{n}_{x} \mathrm{~d} l\right| \leq\left\|u_{x x}\right\|_{0, \partial \Omega}\left\|r_{h}\right\|_{0, \partial \Omega} \leq C h^{-1 / 2}\|u\|_{3, \Omega}\left\|r_{h}\right\|_{0, \Omega} .
$$

We establish (3.21) from (3.22) and (3.25)-(3.28). 


\subsection{Superconvergence}

If the solution $(\mathbf{u}, p)$ in (2.12) has more regularity $(\mathbf{u}, p) \in\left[H^{2}(\Omega)\right]^{2} \times H^{1}(\Omega)$, then, since $-\Delta \mathbf{u}+\nabla p=\mathbf{f}$, $(\mathbf{u}, p)$ satisfies the following:

$$
B_{h}(\mathbf{u}, p ; \mathbf{v}, q)=(\mathbf{f}, \mathbf{v})+\sum_{Q \in \mathcal{T}_{h}}\left(\mathbf{f}, \tau_{Q} \nabla q\right)_{Q}, \quad \forall(\mathbf{v}, q) \in\left[H_{0}^{1}(\Omega)\right]^{2} \times M_{h}
$$

For the finite element solution $\left(\mathbf{u}_{h}, p_{h}\right) \in X_{h} \times M_{h}$ in (2.13), we have the following orthogonality, from (3.29) and Lemma 3.1,

$$
B_{h}\left(\mathbf{u}-\mathbf{u}_{L}, p-p_{h} ; \mathbf{v}_{L}, q_{h}\right)=0, \quad \forall\left(\mathbf{v}_{L}, q_{h}\right) \in\left[\mathbf{Q}_{1, h, 0}\right]^{2} \times M_{h},
$$

where $\mathbf{u}_{L}$ is the bilinear part of $\mathbf{u}_{h}$.

We reach at the superconvergence of the pressure $p_{h} \in M_{h}$ in the following theorem.

Theorem 3.6. Let $(\mathbf{u}, p) \in\left[H_{0}^{1}(\Omega)\right]^{2} \times L_{0}^{2}(\Omega),\left(\mathbf{u}_{h}, p_{h}\right) \in X_{h} \times M_{h}$ be the solutions of (2.12), (2.13), respectively. If the solution $(\mathbf{u}, p)$ belongs to $\left[H^{3}(\Omega)\right]^{2} \times H^{2}(\Omega)$, then

$$
\left\|p-p_{h}\right\|_{0} \leq C h^{3 / 2}\left(\|\mathbf{u}\|_{3}+|p|_{2}\right)
$$

for some constant $C$ which depends only on $\Omega$.

Proof. Let $p_{i} \in \mathcal{N C}^{h}(\Omega)$ be the standard interpolation of $p \in H^{2}(\Omega)$ by $(2.1)$ and define $p_{I} \in M_{h}$ as

$$
p_{I}=p_{i}-\oint_{\Omega} p_{i} \mathrm{~d} s
$$

Then, from (2.2), the interpolation error is estimated by

$$
\left\|p-p_{I}\right\|_{0}+h\left(\sum_{Q \in \mathcal{T}_{h}}\left|p-p_{I}\right|_{1, Q}^{2}\right)^{1 / 2} \leq C h^{2}|p|_{2},
$$

for some constant $C$ which depends only on $\Omega$.

For $p_{I}$ and the bilinear interpolant $\mathbf{u}_{I}$ of $\mathbf{u}$, the orthogonality in (3.30) is rewritten as

$$
B_{h}\left(\mathbf{u}-\mathbf{u}_{I}, p-p_{I} ; \mathbf{v}_{L}, q_{h}\right)=B_{h}\left(\mathbf{u}_{L}-\mathbf{u}_{I}, p_{h}-p_{I} ; \mathbf{v}_{L}, q_{h}\right) .
$$

Then, there exists $\left(\mathbf{w}_{L}, r_{h}\right) \in\left[\mathbf{Q}_{1, h, 0}\right]^{2} \times M_{h}$ such that, by (3.32) and Lemma 3.2,

$$
\begin{aligned}
\left\|\left(\mathbf{u}_{L}-\mathbf{u}_{I}, p_{h}-p_{I}\right)\right\|\left\|_{B}||\left(\mathbf{w}_{L}, r_{h}\right)\right\| \|_{B} & \leq \frac{1}{\beta} B_{h}\left(\mathbf{u}_{L}-\mathbf{u}_{I}, p_{h}-p_{I} ; \mathbf{w}_{L}, r_{h}\right) \\
& =\frac{1}{\beta} B_{h}\left(\mathbf{u}-\mathbf{u}_{I}, p-p_{I} ; \mathbf{w}_{L}, r_{h}\right),
\end{aligned}
$$

with some constant $\beta$, since $\left(\mathbf{u}_{L}-\mathbf{u}_{I}, p_{h}-p_{I}\right) \in\left[\mathbf{Q}_{1, h, 0}\right]^{2} \times M_{h}$.

From the definition of $B_{h}$, we have

$$
\begin{aligned}
B_{h}\left(\mathbf{u}-\mathbf{u}_{I}, p-p_{I} ; \mathbf{w}_{L}, r_{h}\right)= & \left(\nabla\left(\mathbf{u}-\mathbf{u}_{I}\right) \cdot \nabla \mathbf{w}_{L}\right)+\left(r_{h}, \operatorname{div}\left(\mathbf{u}-\mathbf{u}_{I}\right)\right) \\
& -\left(p-p_{I}, \operatorname{div} \mathbf{w}_{L}\right)+\sum_{Q \in \mathcal{T}_{h}}\left(\nabla\left(p-p_{I}\right), \tau_{Q} \nabla r_{h}\right)_{Q}-\sum_{Q \in \mathcal{T}_{h}}\left(\Delta\left(\mathbf{u}-\mathbf{u}_{I}\right), \tau_{Q} \nabla r_{h}\right)_{Q} .
\end{aligned}
$$


We apply the superclosenesses in Lemmas 3.3 and 3.5 for the first two terms in the right hand side of (3.34) to get,

$$
\begin{gathered}
\left(\nabla\left(\mathbf{u}-\mathbf{u}_{I}\right) \cdot \nabla \mathbf{w}_{L}\right) \leq C h^{2}|\mathbf{u}|_{3} \quad\left\|\left(\mathbf{w}_{L}, r_{h}\right)\right\| \|_{B}, \\
\left(r_{h}, \operatorname{div}\left(\mathbf{u}-\mathbf{u}_{I}\right)\right) \leq C h^{3 / 2}\|\mathbf{u}\|_{3}\left\|\mid\left(\mathbf{w}_{L}, r_{h}\right)\right\| \|_{B} .
\end{gathered}
$$

The third term is simply done by (3.31) into

$$
\left|\left(p-p_{I}, \operatorname{div} \mathbf{w}_{L}\right)\right| \leq C h^{2}|p|_{2}||\left|\left(\mathbf{w}_{L}, r_{h}\right)\right|||_{B} .
$$

With (3.3) and (3.31), the fourth term is estimated by

$$
\begin{aligned}
\left|\sum_{Q \in \mathcal{T}_{h}}\left(\nabla\left(p-p_{I}\right), \tau_{Q} \nabla r_{h}\right)_{Q}\right| & =\left|\sum_{Q \in \mathcal{T}_{h}}\left(\tau_{Q}^{1 / 2} \nabla\left(p-p_{I}\right), \tau_{Q}^{1 / 2} \nabla r_{h}\right)_{Q}\right| \\
& \leq \gamma C h^{2}|p|_{2}||\left|\left(\mathbf{w}_{L}, r_{h}\right)\right| \|_{B} .
\end{aligned}
$$

For the last term in the right hand side of (3.34), let $\overline{\Delta \mathbf{u}} \in\left[P_{h}\right]^{2}$ be a piecewise constant interpolation of $\Delta \mathbf{u}$ such that

$$
\int_{Q} \Delta \mathbf{u} \mathrm{d} s=\int_{Q} \overline{\Delta \mathbf{u}} \mathrm{d} s, \quad \text { for all } Q \text { in } \mathcal{T}_{h},
$$

whose interpolation error is estimated by

$$
\|\Delta \mathbf{u}-\overline{\Delta \mathbf{u}}\|_{0} \leq C h|\mathbf{u}|_{3} .
$$

Then, by (3.3) and (3.38), we have,

$$
\begin{aligned}
\left(\Delta\left(\mathbf{u}-\mathbf{u}_{I}\right), \tau_{Q} \nabla r_{h}\right)_{Q} & =\left(\Delta \mathbf{u}-\overline{\Delta \mathbf{u}}, \tau_{Q} \nabla r_{h}\right)_{Q}+\left(\overline{\Delta \mathbf{u}}, \tau_{Q} \nabla r_{h}\right)_{Q} \\
& =\left(\tau_{Q}^{1 / 2}(\Delta \mathbf{u}-\overline{\Delta \mathbf{u}}), \tau_{Q}^{1 / 2} \nabla r_{h}\right)_{Q}+\alpha^{2} h^{2}\left(\Delta \mathbf{u}, \nabla r_{h}\right)_{Q},
\end{aligned}
$$

since $\Delta \mathbf{u}_{I}$ vanishes and $\nabla r_{h}$ is a constant vector.

By (3.3) and (3.39), the sum of the first terms in (3.40a) and (3.40b) for all $Q \in \mathcal{T}_{h}$ is estimated by

$$
\left|\sum_{Q \in \mathcal{T}_{h}}\left(\tau_{Q}^{1 / 2}(\Delta \mathbf{u}-\overline{\Delta \mathbf{u}}), \tau_{Q}^{1 / 2} \nabla r_{h}\right)_{Q}\right| \leq \gamma C h^{2}|\mathbf{u}|_{3}||\left|\left(\mathbf{w}_{L}, r_{h}\right)\right| \|_{B} .
$$

For the second term, through integration by parts and since div $\mathbf{u}$ vanishes, we have:

$$
\begin{aligned}
\sum_{Q \in \mathcal{T}_{h}}\left(\Delta \mathbf{u}, \nabla r_{h}\right)_{Q} & =\sum_{Q \in \mathcal{T}_{h}}<\Delta \mathbf{u} \cdot \mathbf{n}, r_{h}>_{\partial Q}-\sum_{Q \in \mathcal{T}_{h}}\left(\operatorname{div} \Delta \mathbf{u}, r_{h}\right)_{Q} \\
& =\sum_{Q \in \mathcal{T}_{h}}<\Delta \mathbf{u} \cdot \mathbf{n}, r_{h}>_{\partial Q} .
\end{aligned}
$$

Applying Proposition 3.4 for the edges in the interior of $\Omega$, we obtain

$$
\left|\sum_{Q \in \mathcal{T}_{h}}\left(\Delta \mathbf{u}, \nabla r_{h}\right)_{Q}\right| \leq C|\Delta \mathbf{u}|_{1}||\left|\left(\mathbf{w}_{L}, r_{h}\right)\right|||_{B}+\left|<\Delta \mathbf{u} \cdot \mathbf{n}, r_{h}>_{\partial \Omega}\right| .
$$


TABLE 1. Error table for uniform rectangular meshes (M1).

\begin{tabular}{lcccccc}
\hline \multirow{2}{*}{ Mesh } & \multicolumn{2}{c}{$\left\|\mathbf{u}-\mathbf{u}_{h}\right\|_{0}$} & \multicolumn{2}{c}{$\left\|\nabla \mathbf{u}-\nabla \mathbf{u}_{h}\right\|_{0}$} & \multicolumn{2}{c}{$\left\|p-p_{h}\right\|_{0}$} \\
\cline { 2 - 7 } & Value & Order & Value & Order & Value & Order \\
\hline $16 \times 16$ & $2.2187 \mathrm{E}-3$ & & $1.8359 \mathrm{E}-1$ & & $5.7334 \mathrm{E}-2$ & \\
$32 \times 32$ & $5.2254 \mathrm{E}-4$ & 2.0861 & $8.8997 \mathrm{E}-2$ & 1.0447 & $2.3560 \mathrm{E}-2$ & 1.2830 \\
$64 \times 64$ & $1.2736 \mathrm{E}-4$ & 2.0367 & $4.4001 \mathrm{E}-2$ & 1.0162 & $9.6639 \mathrm{E}-3$ & 1.2857 \\
$128 \times 128$ & $3.1456 \mathrm{E}-5$ & 2.0175 & $2.1889 \mathrm{E}-2$ & 1.0074 & $3.5357 \mathrm{E}-3$ & 1.4506 \\
$256 \times 256$ & $7.8178 \mathrm{E}-6$ & 2.0085 & $1.0917 \mathrm{E}-2$ & 1.0035 & $1.1435 \mathrm{E}-3$ & 1.6285 \\
$512 \times 512$ & $1.9491 \mathrm{E}-6$ & 2.0039 & $5.4530 \mathrm{E}-3$ & 1.0015 & $3.5626 \mathrm{E}-4$ & 1.6825 \\
$1024 \times 1024$ & $4.8663 \mathrm{E}-7$ & 2.0019 & $2.7253 \mathrm{E}-3$ & 1.0007 & $1.1460 \mathrm{E}-4$ & 1.6363 \\
\hline
\end{tabular}

TABLE 2. Error table for non-uniform bisection mesh (M2).

\begin{tabular}{lcccccc}
\hline \multirow{2}{*}{ mesh } & \multicolumn{2}{c}{$\left\|\mathbf{u}-\mathbf{u}_{h}\right\|_{0}$} & \multicolumn{2}{c}{$\left\|\nabla \mathbf{u}-\nabla \mathbf{u}_{h}\right\|_{0}$} & \multicolumn{2}{c}{$\left\|p-p_{h}\right\|_{0}$} \\
\cline { 2 - 7 } & value & order & value & order & value & order \\
\hline $16 \times 16$ & $2.6092 \mathrm{E}-3$ & & $1.9955 \mathrm{E}-1$ & & $6.1404 \mathrm{E}-2$ & \\
$32 \times 32$ & $6.1353 \mathrm{E}-4$ & 2.0884 & $9.6495 \mathrm{E}-2$ & 1.0482 & $2.4388 \mathrm{E}-2$ & 1.3322 \\
$64 \times 64$ & $1.4974 \mathrm{E}-4$ & 2.0347 & $4.7694 \mathrm{E}-2$ & 1.0167 & $9.8453 \mathrm{E}-3$ & 1.3087 \\
$128 \times 128$ & $3.7029 \mathrm{E}-5$ & 2.0157 & $2.3729 \mathrm{E}-2$ & 1.0072 & $3.5883 \mathrm{E}-3$ & 1.4561 \\
$256 \times 256$ & $9.2103 \mathrm{E}-6$ & 2.0073 & $1.1837 \mathrm{E}-2$ & 1.0033 & $1.1704 \mathrm{E}-3$ & 1.6164 \\
$512 \times 512$ & $2.2979 \mathrm{E}-6$ & 2.0029 & $5.9127 \mathrm{E}-3$ & 1.0014 & $3.7041 \mathrm{E}-4$ & 1.6598 \\
$1024 \times 1024$ & $5.7649 \mathrm{E}-7$ & 1.9949 & $2.9551 \mathrm{E}-3$ & 1.0006 & $1.2099 \mathrm{E}-4$ & 1.6142 \\
\hline
\end{tabular}

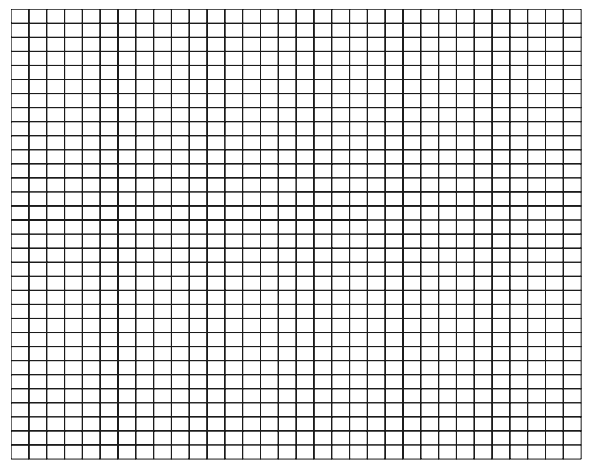

FIgURE 1. $32 \times 32$ Uniform rectangular mesh (M1).

Then, the global trace theorem for $\Delta \mathbf{u}$ and the local one in (3.14) for $r_{h}$ establish

$$
\left|<\Delta \mathbf{u} \cdot \mathbf{n}, r_{h}>_{\partial \Omega}\right| \leq C h^{-1 / 2}\|\Delta \mathbf{u}\|_{1}\left\|\mid\left(\mathbf{w}_{L}, r_{h}\right)\right\| \|_{B} .
$$

Combining (3.33a)-(3.37) and (3.40a)-(3.43), we have

$$
\left\|\left|\left(\mathbf{u}_{L}-\mathbf{u}_{I}, p_{h}-p_{I}\right)\right|\right\|_{B} \leq C h^{3 / 2}\left(\|\mathbf{u}\|_{3}+|p|_{2}\right),
$$

which completes the proof through (3.31) and the definition of the triple norm in (3.7).

\section{Numerical RESUlts}

For the test problem, we chose the stream function $\phi$ on $\Omega=(0,1)^{2}$ such that

$$
\phi(x, y)=s(x) s(y), \quad \text { for } s(t)=\sin (2 \pi t)\left(t^{2}-t\right),
$$




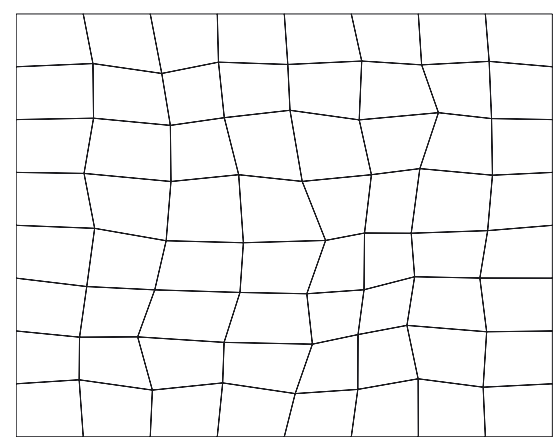

(a) $8 \times 8$ initial non-uniform mesh

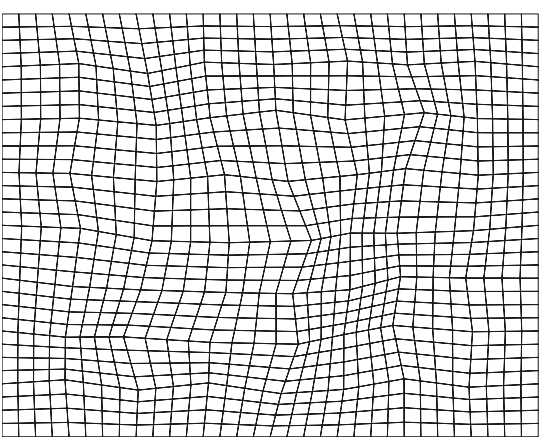

(b) $32 \times 32$ non-uniform mesh by bisecting

Figure 2. Non-uniform meshes by recursive bisecting from $8 \times 8$ mesh (M2).

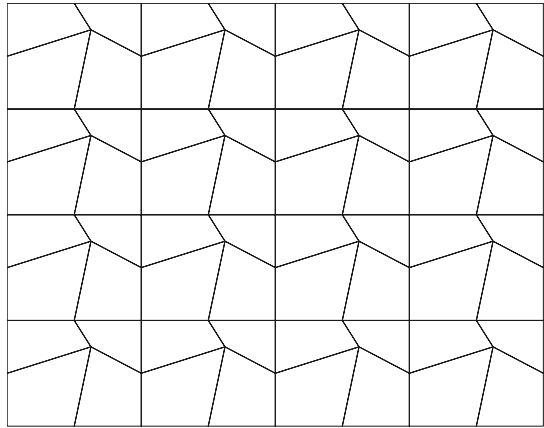

(a) $8 \times 8$ zigzag mesh

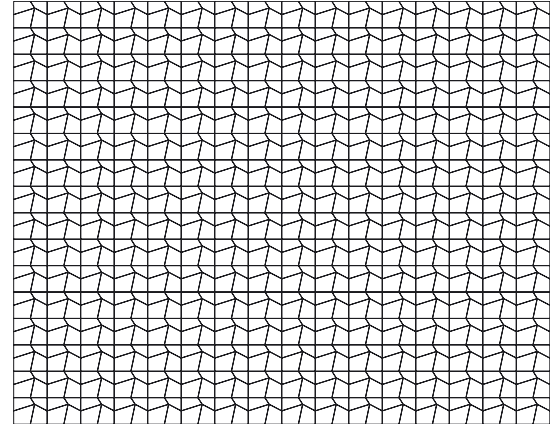

(b) $32 \times 32$ zigzag mesh

Figure 3. Zigzag meshes (M3).

TABLE 3. Error table for zigzag meshes (M3).

\begin{tabular}{lcccccc}
\hline \multirow{2}{*}{ Mesh } & \multicolumn{2}{c}{$\left\|\mathbf{u}-\mathbf{u}_{h}\right\|_{0}$} & \multicolumn{2}{c}{$\left\|\nabla \mathbf{u}-\nabla \mathbf{u}_{h}\right\|_{0}$} & \multicolumn{2}{c}{$\left\|p-p_{h}\right\|_{0}$} \\
\cline { 2 - 7 } & Value & Order & Value & Order & Value & Order \\
\hline $16 \times 16$ & $5.0339 \mathrm{E}-3$ & & $2.8134 \mathrm{E}-1$ & & $1.4795 \mathrm{E}-1$ & \\
$32 \times 32$ & $1.2634 \mathrm{E}-3$ & 1.9944 & $1.4068 \mathrm{E}-1$ & 0.9999 & $7.5569 \mathrm{E}-2$ & 0.9692 \\
$64 \times 64$ & $3.1487 \mathrm{E}-4$ & 2.0044 & $7.0199 \mathrm{E}-2$ & 1.0029 & $3.7558 \mathrm{E}-2$ & 1.0087 \\
$128 \times 128$ & $7.8388 \mathrm{E}-5$ & 2.0061 & $3.5027 \mathrm{E}-2$ & 1.0030 & $1.8444 \mathrm{E}-2$ & 1.0260 \\
$256 \times 256$ & $1.9551 \mathrm{E}-5$ & 2.0034 & $1.7489 \mathrm{E}-2$ & 1.0020 & $9.0836 \mathrm{E}-3$ & 1.0218 \\
$512 \times 512$ & $4.8839 \mathrm{E}-6$ & 2.0011 & $8.7357 \mathrm{E}-3$ & 1.0014 & $4.4880 \mathrm{E}-3$ & 1.0172 \\
$1024 \times 1024$ & $1.1883 \mathrm{E}-6$ & 2.0391 & $4.3573 \mathrm{E}-3$ & 1.0035 & $2.1831 \mathrm{E}-3$ & 1.0397 \\
\hline
\end{tabular}

and the velocity $\mathbf{u}$ and pressure $p$ as

$$
\mathbf{u}(x, y)=\operatorname{curl} \phi, \quad p(x, y)=\sin (2 \pi x)\left(\frac{1}{25-10 \tan ^{2} y}+\frac{3}{10}\right) .
$$

Solving (2.13) with the source function $\mathbf{f}=-\Delta \mathbf{u}+\nabla p$, we obtained $\left(\mathbf{u}_{h}, p_{h}\right) \in X_{h} \times M_{h}$.

The numerical results are shown in Tables 1 and 2 for uniform rectangular(M1) and non-uniform bisection(M2) meshes as depicted in Figures 1 and 2, respectively. The non-uniform bisection meshes are obtained by recursive bisecting from the $8 \times 8$ initial non-uniform mesh in Figure 2a. 
We can observe the expected order of convergence for the velocity in (2.14). For the pressure, the superconvergence of more order than $3 / 2$ analyzed in Theorem 3.6 appears in uniform rectangular and even non-uniform bisection meshes.

For the zigzag meshes (M3) as in Figure 3, the numerical results in Table 3 does not show superconvergence in pressure any more.

Acknowledgements. This research was partially supported by Basic Science Research Program through the National Research Foundation of Korea (NRF) funded by the Ministry of Education, Science and Technology (2012R1A1A2009509). This paper also resulted from the Konkuk University research support program.

\section{REFERENCES}

[1] D.N. Arnold, F. Brezzi and M. Fortin, A stable finite element for the Stokes equations. CALCOLO 21 (1984) $337-344$.

[2] I. Babuška, The finite element method with Lagrange multipliers. Numer. Math. 20 (1973) 179-192.

[3] W. Bai, The quadrilateral 'Mini' finite element for the Stokes problem. Comput. Methods Appl. Mech. Eng. 143 (1997) 41-47.

[4] F. Brezzi, On the existence, uniqueness and approximation of saddle point problems arising from Lagrangian multipliers. RAIRO Anal. Numer. R2 8 (1974) 129-151.

[5] J. Douglas Jr., J.E. Santos, D. Sheen and X. Ye, Nonconforming Galerkin methods based on quadrilateral elements for second order elliptic problems. RAIRO: M2AN 33 (1999) 747-770.

[6] H. Eichel, L. Tobiska and H. Xie, Supercloseness and superconvergence of stabilized low order finite element discretization of the Stokes Problem. Math. Comput. 80 (2011) 697-722.

[7] L.P. Franca, S.P. Oliveira and M. Sarkis, Continuous Q1-Q1 Stokes elements stabilized with non-conforming null edge average velocity functions. Math. Models Meth. Appl. Sci. 17 (2007) 439-459.

[8] V. Girault and P.A. Raviart, Finite element methods for the Navier-Stokes equations: Theory and Algorithms. Springer-Verlag, New York (1986).

[9] C. Park and D. Sheen, $P_{1}$-nonconforming quadrilateral finite element methods for second-order elliptic problems. SIAM J. Numer. Anal. 41 (2003) 624-640.

[10] R. Rannacher and S. Turek, Simple nonconforming quadrilateral Stokes element. Numer. Methods Partial Differ. Eq. 8 (1992) 97-111. 